MPI-Ph/93-87

AZPH-TH/93-33

November 1993

\title{
Super-Instantons and the Reliability of Perturbation Theory in Non-Abelian Models
}

\section{Adrian Patrascioiu}

Physics Department and Center for the Study of Complex Systems University of Arizona, Tucson, AZ 85721, U.S.A.

and

\section{Erhard Seiler}

Max-Planck-Institut für Physik

- Werner-Heisenberg-Institut -

Föhringer Ring 6, 80805 Munich, Germany

\begin{abstract}
In dimension $D \leq 2$ the low temperature behavior of systems enjoying a continuous symmetry is dominated by super-instantons: classical configurations of arbitrarily low energy. Perturbation theory in the background of a super-instanton produces thermodynamic answers for the invariant Green's functions that differ from the standard ones, but only in non-Abelian models and only starting at $O\left(1 / \beta^{2}\right)$. This effect modifies the $\beta$-function of the $O(N)$ models and persists in the large $N$ limit of the $O(N)$ models.
\end{abstract}


Consider a $\mathbf{Z}^{D}$ lattice and let $\Lambda$ be some given finite subset of it. The non-linear $O(N) \sigma$ model with standard nearest neighbor interaction (s.n.n.i.) at inverse temperature $\beta$ is defined by the following partition function $Z$ :

$$
Z=\int \prod_{\langle i j\rangle} \exp \left(\beta S_{i} \cdot S_{j}\right) \prod_{i}\left(\delta\left(S_{i}^{2}-1\right) d S_{i}\right)
$$

Here $\mathrm{S}$ is an $N$-vector, the integration is over the spins inside $\Lambda$ with some boundary conditions (b.c.).

We shall be interested in the behavior of fixed (lattice) distance Green's functions, such as the expectation value of the energy density $\langle S(0) \cdot S(1)\rangle$, at large $\beta$. As $\beta \rightarrow \infty$, this finite system will 'freeze' into the one or several configurations which minimize the energy (at given b.c.). For $\beta$ large but finite, the spins will fluctuate mildly around one of these classical configurations and thus one can compute the Green's functions by a standard saddle point expansion. This perturbation theory (PT) approach produces the correct asymptotic expansion in $1 / \beta$; more precisely, there exists an infinite set of finite numbers $\left\{d_{k}\right\}$ such that for $\beta$ sufficiently large

$$
\left|\langle S(0) \cdot S(1)\rangle-\sum_{j=0}^{k} \frac{c_{j}}{\beta^{j}}\right|<\frac{d_{k+1}}{\beta^{k+1}}
$$

Here $\left\{c_{k}\right\}$ are the expansion coefficients produced by PT.

Of course both $\left\{c_{k}\right\}$ and $\left\{d_{k}\right\}$ depend upon the linear size $L$ of $\Lambda$ and the b.c. used. In particular, they could diverge as $L \rightarrow \infty$. But it could also happen that even though the coefficients $c_{k}$ have finite limits, for all $k>k_{o}$ the left hand side of eq.(2) is not $o\left(\beta^{-k}\right)$ uniformly in $L$, i.e. if multiplied by $\beta^{k}$ it is not bounded by a function of $\beta$ alone that vanishes in the limit $\beta \rightarrow \infty$. This would indicate that the $L \rightarrow \infty$ limits of the $c_{k}$ are not the coefficients of the correct asymptotic expansion in that limit. In other words, PT itself cannot be used to establish its correctness in producing the asymptotic expansion for $\langle S(0) \cdot S(1)\rangle$ at large $\beta$. For that purpose one needs bounds uniform in $L$ on the numbers $\left\{d_{k}(L)\right\}$. This property of PT was established for the Abelian case $O(2)$ [1], but the authors could not extend the proof to the non-Abelian case $O(N) N>2$.

The general attitude in the community has been to ignore this issue. In fact as we tried to emphasize in the past [2, 3] there are some good reasons to expect trouble for $D \leq 2$. Firstly in those cases, the Mermin-Wagner theorem guarantees that the basic assumption underlying a saddle point approximation, the existence of a dominant configuration, is violated as $L \rightarrow \infty$. Secondly in $1 D$, where one can obtain the true answer using the transfer matrix formalism, one can see explicitely that for $N>2$, the coefficient of $1 / \beta^{2}$ in the expansion of $\langle S(0) \cdot S(1)\rangle$ is finite yet incorrectly given by PT; that means that if one truncated the series at this order, the remainder would be $O\left(1 / \beta^{2}\right)$, rather 
than $o\left(1 / \beta^{2}\right)$, as required by a putative asymptotic expansion.

In this letter we would like to point out that also in $2 D$, the two limits $\beta \rightarrow \infty$ and $L \rightarrow \infty$ do not commute for the non-Abelian case $N>2$. The idea is the following: let $\Lambda$ be a square of size $L \times L$ and on its edge freeze the spins along the $x$-axis. Since by the Mermin-Wagner theorem the infinite volume Gibbs state is $O(N)$ symmetric, for $L \rightarrow \infty$, the expectation value of $\langle S(0) \cdot S(1)\rangle$ should be unchanged if the spin at the origin $S(0)$ is also frozen in some given direction. So if one believes in PT, one should also expect that the PT coefficients approach values independent of whether $S(0)$ has been frozen or not. As it will be seen, this is precisely what happens for $O(2)$ but not for $O(N) N>2$ starting at $O\left(1 / \beta^{2}\right)$.

First let us discuss the massless Gaussian, described by the partition function

$$
Z=\int \prod_{\langle i j\rangle} \exp \left(-\frac{1}{2}\left(\varphi_{i}-\varphi_{j}\right)^{2}\right) \prod_{i} d \varphi_{i}
$$

It too possesses a continuous symmetry, which in $2 D$ cannot be broken spontaneously. Consequently, if we fix $\varphi=0$ at the edge of the square and measure an invariant observable, such as $D(0,1)=(\varphi(0)-\varphi(1))^{2}$, for $L \rightarrow \infty$ the result should become independent of whether $\varphi(0)$ is fixed or not. In particular even if $\varphi(0)=0, D(0,1)$ should converge to $1 / 2$ for $L \rightarrow \infty$.

This is clearly the case, as it can be seen from the data in Tab.1 (line 1). These data were produced by a Monte Carlo study of this model using the overrelaxation procedure. Besides $D(0,1)$ we measured certain Green's functions of the massless Gaussian with these b.c., which will be needed to compute the PT expansion of $\langle S(0) \cdot S(1)\rangle$ in $O(N)$ - see below. We increased the number of measurements with $L$; as an example, for $L=80$ we took 100, 000 measurements, separated by 5 sweeps of the lattice. Similarly, the number of thermalization sweeps increased with $L$, reaching 10,000 for $L=80$. We estimated the error by a standard binning procedure.

Let us return now to the case of interest of the $O(N)$ models. Firstly, although it is obvious, we verified that as we increase $L,\langle S(0) \cdot S(1)\rangle$ does become independent of what we do with $S(0)$. We studied both $O(2)$ and $O(3)$. The spins at the edge of the square were fixed along the $x$-axis. The spin at the origin $S(0)$ was either free ('Dirichlet b.c.') or also frozen along the $x$-axis ('super-instanton b.c.'). We performed a Monte Carlo study using a cluster algorithm. Some typical results are shown in Tab.2, where we give also the value of $\langle S(0) \cdot S(1)\rangle$ at the same $\beta$ using periodic b.c. The results indicate that $\langle S(0) \cdot S(1)\rangle$ does become independent of the b.c. as $L \rightarrow \infty$, although the approach is slower for the super-instanton b.c..

Next let us see what PT would predict for super-instanton b.c. Using the standard parametrization 


$$
S_{i}=\left(\pi_{i}, \sqrt{1-\pi_{i}^{2}}\right)
$$

then expanding in $\pi$, one obtains

$$
\begin{aligned}
\langle S(0) \cdot S(1)\rangle & =1-\frac{(N-1)}{2 \beta} G(1,1) \\
& -\frac{1}{\beta^{2}}(N-1)^{2}\left[\frac{\left(G(1,1)^{2}\right.}{8}-\frac{t}{4}\right] \\
& +(N-1)\left[-\frac{G(1,1)^{2}}{4}+\frac{v}{2}\right]+\ldots
\end{aligned}
$$

where

$$
\begin{aligned}
G(i, j) & =\langle\pi(i) \cdot \pi(j)\rangle \\
t & =\sum_{<i, j>}\left[G(1, i)^{2}-G(1, j)^{2}\right][G(i, i)-G(j, j)] \\
v & =\sum_{<i, j>}[G(1, i)-G(1, j)]\{G(1, j)[G(i, j)-G(j, j)] \\
& -G(1, i)[G(i, j)-G(i, i)]\}
\end{aligned}
$$

The super-instanton b.c. on $S(\pi=0$ along the edge and at 0$)$, imply that $G(i, j)$ in eq.(6) is precisely the Green's function of the massless Gaussian studied above. From that study we already know that the term of $O(1 / \beta)$ will converge to a value independent of the b.c. as $L \rightarrow \infty$. To study the term of $O\left(1 / \beta^{2}\right)$ we computed $t$ and $v$ numerically and report the values obtained in Tab.1.

To interprete these data, let us recall that if instead of the super-instanton b.c. one would use periodic b.c., one would obtain [4

$$
\langle S(0) \cdot S(1)\rangle=1-\frac{N-1}{\beta} D(1)-\frac{N-1}{2 \beta^{2}} D(1)^{2}+\ldots
$$

Here $D(1)=\frac{1}{2 D}\left(1-\frac{1}{L^{2}}\right)$ and we have neglected a term of $O\left(1 / \beta^{2}\right)$ coming from the elimination of the zero mode which vanishes very rapidly with increasing $L$. Since eq.(5) contains a term proportional to $(N-1)^{2}$, absent in eq. (7), the two would agree only if $t$ converged to $1 / 8$. In fact our data presented in Tab. 1 suggest that $t$ converges to 0.035 or less. On the contrary, agreement of eqs.(5) and (7) for $N=2$ requires that $v-t / 2$ converges to $1 / 8$ and our data certainly seem consistent with that.

The fact that $v-t / 2$ goes to $1 / 8$ can also be proven analytically. It follows after some algebraic manipulations that 
$v-t / 2=\sum_{<i, j>}\left(-G(i, j)+\frac{1}{2} G(i, i)+\frac{1}{2} G(j, j)\right)(G(0, i)-G(1, i)-G(0, j)+G(1, j))$

It can be justified to take the limit $L \rightarrow \infty$ under the sum, and then it is seen easily by a summation by parts that it becomes $1 / 8$. On the other hand, the same procedure is not justified for the sums defining $v$ and $t$ separately (because the sums are not convergent uniformly in $L$ ); one would obtain $t=$ $1 / 8$ and $v=3 / 16$, which would agree with Hasenfratz's formula (7), but clearly disagrees with our data.

Therefore our results suggest that if in $2 D$ one takes the limit $L \rightarrow \infty$ termwise in the PT expansion, the result depends upon the b.c. used, but only for non-Abelian models and then only starting at $O\left(1 / \beta^{2}\right)$. Since the correct asymptotic expansion has to be independent of b.c. by the Mermin-Wagner theorem, one must conclude that taking $L \rightarrow \infty$ termwise is not a legitimate procedure. Of course it could happen that in $2 D$ this procedure gives the correct infinite volume answer for certain b.c., just as it does in $1 D$ if one uses free b.c.. For instance it has been argued that periodic b.c. must be giving the correct infinite volume answer because they agree with the $1 / N$ expansion. Unfortunately, as we explained before [3], this argument is not correct because it involves again an a priori illegitimate interchange of two limits: $\beta \rightarrow \infty$ and $N \rightarrow \infty$. So while it may happen that in $2 D$ periodic b.c. do give the correct infinite volume PT coefficients, to our knowledge there is no support for that belief at the present time.

We also computed the PT coefficients to the 2-point function at distances $x$ larger than one lattice unit, and found that with super-instanton b.c. one obtains a different answer in the thermodynamic limit for $N>2$. Since for large $x(x>4)$ this modification grows with $x$ like $G(x, x)^{2}$, it will give rise to a $\beta$-function whose leading coefficient is different from the one found in the literature (obtained by using PT with periodic b.c.). This fact casts doubt upon the alleged asymptotic freedom of the $O(N) N>2$ models. Details will be reported elsewhere.

Over the years it has been argued that in theories which are perturbatively asymptotically free, PT should be applicable at short distances, where the system is well ordered. In fact starting at $O\left(1 / \beta^{2}\right)$, as it can be seen from eqs. (5) and (6), a PT computation involves sums over all distances. Consequently our finding that the trouble starts only from $O\left(1 / \beta^{2}\right)$ is quite understandable. The trouble does not arise in $O(2)$ because there, by parametrizing the spin as $(\cos (\varphi), \sin (\varphi))$, the Gibbs measure becomes a function of $\nabla \varphi$ only.

Before concluding let us explain why we called these b.c. super-instanton b.c.. Let $\Lambda$ be (the closest lattice domain to) a disk of radius R. Let us use the following spin parametrization 


$$
S_{i}=\left(\pi_{i}, \sqrt{1-\pi_{i}^{2}} \sin \left(\varphi_{i}\right), \sqrt{1-\pi_{i}^{2}} \cos \left(\varphi_{i}\right)\right)
$$

where the $\pi_{i}$ are now $(N-2)$-vectors. We choose as b.c. $\pi=0$ along the edge and at 0 , while $\varphi(0)=0$ but $\varphi=c>0$ along the edge. To do PT we need first the configuration of minimal energy with these b.c.: it is some lattice version of the following continuum expression

$$
\begin{aligned}
& \varphi(r, \theta)=c \log r / \log R \\
& \pi(r, \theta)=0
\end{aligned}
$$

Please notice that this classical configuration has a vanishing energy as $R \rightarrow$ $\infty$, hence the name super-instanton.

More precisely, twenty years ago Kosterlitz and Thouless (KT) [5] argued that at large $\beta$ the partition function is dominated by the classical configurations, which have since been called instantons. According to KT in $O(2)$ the dominant configurations are vortices and their energy is actually $O(\log (R))$ (hence they are bound), while in $O(3)$ they are 'hedgehogs' of energy $O\left(R^{0}\right)$, which create exponential decay no matter how large $\beta$ is. In fact, as we see now, the super-instantons are the dominant configurations in all $O(N)$ models, since their energy vanishes for $R \rightarrow \infty$. They occur for all $D \leq 2$ and are the 'enforcers' of the Mermin-Wagner theorem (a related idea underlies Pfister's [6] proof of that theorem): Let us for simplicity work in the continuum, with $a$ playing the role of an UV cutoff, and use the fact that the configuration of minimal energy has a $\varphi$ that only depends on the radius $r$. Then

$$
\begin{aligned}
c & =\int_{a}^{R} d r \frac{d}{d r} \varphi \\
E & =2 \pi \int_{a}^{R} d r r^{D-1}\left(\frac{d}{d r} \varphi\right)^{2} .
\end{aligned}
$$

With the scalar product

$$
(f, g)=\int_{a}^{R} d r r^{D-1} f^{*} g
$$

we obtain

$$
c=\left(r^{1-D}, \frac{d}{d r} \phi\right)
$$

and by Schwarz's inequality

$$
c \leq\left\|r^{1-D}\right\| \sqrt{E}=\sqrt{\frac{R^{2-D}-a^{2-D}}{2-D}} \sqrt{E}
$$


This gives a lower bound for $E$ that goes to 0 as $R \rightarrow \infty$, whereas for $D>2$ it remains bounded away from 0 . Therefore one can have super-instantons $\left(E \rightarrow 0\right.$ yet $c \geq c_{o}>0$ ) only for $D \leq 2$.

The arguments above suggest that at low temperature, the typical configuration in the $O(N)$ model in dimension $D \leq 2$ is a gas (or liquid) of super-instantons. The remarkable fact is that we had reached the same conclusion two years ago, when we investigated these models by mapping them into a certain percolation problem [7, 8]; namely if we consider a certain patch

of the sphere $S^{N-1}$ of angular opening $O(1 / \sqrt{\beta})$ and ask what is its inverse image in a typical configuration, our percolation arguments indicated that the answer must be that it forms rings of arbitrarily large size (more precisely, neither the inverse image of the patch nor of its complement percolates). This super-instanton like structure was found to be responsible for the absence of exponential decay in all $O(N)$ models at $\beta$ sufficiently large. Now we see that it may also be responsible for the failure of PT in non-Abelian models. Indeed imagine doing PT in the background of a super-instanton. Even though the new couplings induced by this external field vanish as $1 /(\log R)^{2}$, the potential IR divergences present in PT could produce nonvanishing effects as $R \rightarrow \infty$. In fact the effect could happen only for $N>2(N=2$ involves only $\nabla \phi$, which is IR finite) and only starting at $O\left(1 / \beta^{2}\right)$ since we must compensate two powers of $1 / \log R$ - this is in agreement with the findings reported above, which represented PT in the background of the trivial super-instanton.

A.P. would like to acknowledge the hospitality of the IPN of the Université de Paris Sud and of the Werner-Heisenberg-Institute.

\section{References}

[1] J.Bricmont, J.-R.Fontaine, J.L.Lebowitz, E.H.Lieb and T.Spencer, Commun.Math.Phys. 78 (1981) 545. Academic Press, London - New York 1972.

[2] A.Patrasciou and J.-L. Richard, Phys. Lett. 149B (1984) 167.

[3] A.Patrascioiu and E.Seiler, The Difference between Abelian and NonAbelian Models: Fact and Fancy, preprint MPI-Ph/91-88.

[4] P.Hasenfratz, Phys.Lett.B141 (1984)385. New York etc. 1989.

[5] J.M.Kosterlitz and D.J.Thouless, J. Phys. (Paris) 32 (1975) 581.

[6] C.Pfister, Commun.Math.Phys. 79 (1981) 181.

[7] A.Patrascioiu, Existence of Algebraic Decay in non-Abelian Ferromagnets, University of Arizona preprint AZPH-TH/91-49. 
[8] A.Patrascioiu and E.Seiler, Percolation Theory and the Existence of a Soft Phase in 2D Spin Models, Nucl.Phys.B.(Proc. Suppl.) 30 (1993) 184. 
Tab.1: The PT coefficients to order $1 / \beta^{2}$ of $\langle S(0) \cdot S(1)\rangle$ in the s.n.n.i. $O(N)$ models with super-instanton b.c.

\begin{tabular}{|l||l|l|l|l|l|}
\hline$L$ & 10 & 20 & 40 & 60 & 80 \\
\hline \hline$G(1,1)$ & $.3752(10)$ & $.4006(12)$ & $.4143(8)$ & $.4211(13)$ & $.4314(22)$ \\
\hline $8 v-4 t$ & $.657(32)$ & $.709(40)$ & $.772(18)$ & $.792(18)$ & $.833(74)$ \\
\hline $8 t$ & $.345(46)$ & $.296(47)$ & $.305(18)$ & $.284(26)$ & $.317(77)$ \\
\hline
\end{tabular}

Tab.2a: $\langle S(0) \cdot S(1)\rangle$ for the s.n.n.i. $O(2)$ model with periodic (per.), Dirichlet (Dir.) and super-instanton (s.i.) b.c. (Monte Carlo data taken at $\beta=1.1$; the data point for $L=256$ is taken from R. Gupta and C.Baillie, Phys.Rev. B45 (1992) 2883.)

\begin{tabular}{|l||l|l|l|l|}
\hline$L$ & 20 & 40 & 80 & 256 \\
\hline \hline per. & $.7177(6)$ & $.7155(3)$ & $.7146(2)$ & $.7138(1)$ \\
\hline Dir. & $.717(2)$ & $.712(2)$ & & \\
\hline s.i. & $.774(3)$ & $.768(3)$ & $.755(4)$ & \\
\hline
\end{tabular}

Tab.2b: $\langle S(0) \cdot S(1)\rangle$ for the s.n.n.i. $O(3)$ model with periodic (per.), Dirichlet (Dir.) and super-instanton (s.i.) b.c. (Monte Carlo data taken at $\beta=2.0$; the data point for $L=1024$ is taken from J.Apostolakis et al, Phys.Rev. D43 (1991) 268\%.)

\begin{tabular}{|l||l|l|l|l|}
\hline$L$ & 20 & 40 & 80 & 1024 \\
\hline \hline per. & $.7272(5)$ & $.7257(3)$ & $.7253(2)$ & $.72511(3)$ \\
\hline Dir. & $.728(2)$ & $.726(2)$ & & \\
\hline s.i. & $.776(3)$ & $.766(3)$ & $.753(4)$ & \\
\hline
\end{tabular}

